\title{
Climate of change
}

According to the International Panel on Climate Change (IPCC), there is no longer any question that the Earth's climate is warming. The evidence lies in rising average global temperatures, as well as rising sea levels linked to water thermal expansion and widespread melting of snow and ice. The IPCC's latest report, in 2007, concluded that "the observed increase in global average temperatures since the mid-20th century is very likely due to the observed increase in anthropogenic greenhouse gas concentrations."

Not being expert in the relevant science, I accept the IPCC view - at least tentatively - as I have no reason to doubt the integrity or competence of the experts. Some people, however, suspect a perverse scientific conspiracy to brainwash the public or a slavish devotion on the part of scientists to political correctness, or just cannot believe that humans could really have much influence on a system as large as the Earth's climate. Others raise more thoughtful concerns over the soundness of the climate models on which the IPCC has largely based its conclusions and projected scenarios.

The latter point, it seems to me, is indeed legitimate. Given that the Earth's climate is among the most complex systems ever studied by science, it is fair to ask whether our models of it can be trusted. Indeed, are we even attacking the problem in the right way to get the most useful information out of the available observations?

The physical processes influencing global climate include everything from oceanic currents to complex orbital dynamics or terrestrial biochemistry in hundreds of settings. They cover a tremendous range of scales in space and time. Over the past decade or so, efforts to understand climate dynamics have been dominated by computationally intensive modelling aiming to include all these processes, essentially by integrating the equations of motion for the relevant physics, much as one might run a moleculardynamics simulation of a complex fluid flow. Some think this is the only way to gain insight into a system of such overwhelming complexity, and there's certainly no arguing that such work is not important.

In many cases, such models may indeed offer the only way to explore how particular local processes feed up through the vast web of nonlinear interactions to influence climate as a whole. But a number of climate scientists have rightly questioned whether this approach doesn't sometimes lose the big picture for all the details, and whether a coarse-grained modelling approach might not be better for building up basic insight into climate dynamics. Geophysicist Michel Crucifix of the Catholic University of Louvain, Belgium, has presented a strong argument that the latter approach may be fruitful, especially if pursued on the basis of dynamical systems theory, coupled with careful statistical reasoning.

Crucifix explores the idea in the specific context of glacial cycles in the Quaternary period, stretching from about 2.5 million years ago to the present. Over that period, deep-sea sediments and ice-core data show an irregular cycle of cold glacial periods

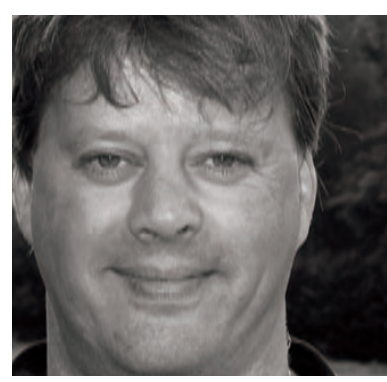

\section{"The most complex model is not necessarily the most useful."}

interspersed with warmer interglacials, with the last glacial period peaking some 19,000 years ago. Geophysicists have been arguing for some time about when the next glacial period should arrive. Working with relatively simple time-series analysis, some suggest that the next glacial is already overdue; others, using numerical climate models of considerable complexity, predict the next glacial in about 50,000 years.

One would naturally expect the more sophisticated model to give the better estimate, although there is, of course, no way to know. However, one might ask whether the same or similar prediction emerges from a less detailed model, even one that tried to reduce climate dynamics to a system of only a few variables. As Crucifix has shown, this is indeed possible.

As he points out, between 1982 and 2002 the late Barry Salzman (a student of Edward Lorenz) and colleagues developed a series of low-dimensional models able to fit past temperature data accurately with only a few parameters. These models considered three physical variables: ice volume, atmospheric carbon dioxide concentration and deep-ocean temperature. Although the models have been criticized for explaining only the data on which they were built, Crucifix argues that this also true for largescale circulation models, which are adjusted on the basis of comparison to past data.

And the low-dimensional models, he argues, can be derived on more legitimate logical grounds. He proposes building models using dynamical systems theory, closely coupled with Bayesian statistical reasoning. Such an approach, he argues, leads to confident estimates not only of model parameters but also of the model form, taking into account model uncertainty stemming from the measurement and interpretation of data.

Following this approach, Crucifix finds a model akin to that of Salzman and colleagues, which gives an accurate account of many features of glacial cycling in the past (while also, it must be said, getting a few things wrong). It also makes the same prediction for the next glaciation $-50,000$ years or so from now - as the more detailed climate model. But this specific result is not as important, I think, as the modelling philosophy behind it.

It is important, yes, to build models that strive to incorporate as much physics detail as possible. But pursuing this strategy alone necessarily risks overlooking the possibility that subsets of climate variables may follow simpler dynamics that can be described by low-dimensional dynamical systems. Such models will never be found unless we look for them.

Crucifix points out that these simpler equations share a qualitative spirit with the irreversible equation for heat flow, introduced originally by Joseph Fourier, at a time when figures such as Laplace and Lagrange championed the power of Newton's reversible equations for particle dynamics. In a sense, Crucifix suggests, Fourier pioneered the idea that complex systems may have simpler descriptions, valid for at least some purposes. Curiously, Fourier later described what we today call the 'greenhouse effect', and argued that the question of the Earth's temperature was one of the most important of natural philosophy.

"The most complex model", Crucifix rightly suggests, "is not necessarily the most useful." The most important matter is learning how to build models that give the best and most useful predictions about climate. Surely that's an idea that makes eminent good sense.

Mark Buchanan 